\title{
Repercussões da nova craniopuntura de yamamoto na dor e qualidade de vida de profissionais de saúde
}

\author{
Repercussions of yamamoto new scalp acupuncture on pain and quality of life of healthcare professionals \\ Repercusiones de nueva craneopuntura de yamamoto sobre dolor y calidad de vida de profesionales de la salud
}

\begin{abstract}
RESUMO
Objetivo: Descrever as repercussões da Nova Craniopuntura de Yamamoto (YNSA) na dor e qualidade de vida de profissionais de saúde. Métodos: Estudo do tipo antes e depois, com profissionais de saúde de uma Estratégia de Saúde da Família, que possuíam dores crônicas. Utilizou-se o instrumento WHOQOL-BREF para avaliar a qualidade de vida antes do início e após o término do tratamento com a YNSA, realizado em um período de 4 semanas. Resultados: Obteve-se melhora estatística dos domínios psicológico, ambientais, de dor, das questões 1 e 2 e do resultado final da qualidade de vida, com destaque para o domínio físico ( $p=0.009$ ). Conclusão: Após o tratamento de YNSA foi possível observar diminuição significativa da dor. Em virtude da melhora da dor também foi possivel observar e comprovar a melhora dos domínios que compõem a qualidade de vida.
\end{abstract}

DESCRITORES: Qualidade de Vida; Dor; Profissionais de Saúde; Acupuntura.

\section{ABSTRACT}

Objective: To describe the repercussions of Yamamoto New Scalp Acupuncture (YNSA) on pain and quality of life of health professionals. Methods: Study before and after, with health professionals of a Family Health Strategy, who had chronic pain. WHOQOL-BREF instrument was used to evaluate quality of life before initiation and after the treatment with the YNSA, performed over a 4-week period. Results: Statistical improvements were obtained in the psychological, environmental, pain domains, questions 1 and 2 , and the final quality of life results, especially the physical domain $(p=0.009)$. Conclusion: After the treatment of YNSA it was possible to observe a significant decrease in pain. Due to the improvement of pain, it was also possible to observe and verify the improvements in the domains that make up the quality of life.

DESCRIPTORS: Quality of Life; Pain; Health Professionals; Acupuncture.

\section{RESUMEN}

Objetivo: Describir las repercusiones de la Nueva Craneopuntura de Yamamoto (YNSA) sobre el dolor y la calidad de vida de los profesionales de la salud. Métodos: Estudio antes y después, con los profesionales de la salud de una Estrategia de salud familiar, que tenían dolor crónico. El instrumento WHOQOL-BREF fue utilizado para evaluar la calidad de vida antes y después del tratamiento con Nueva Craneopuntura de Yamamoto, que se realizó durante un período de 4 semanas. Resultados: Hubo una mejora estadística en los dominios psicológicos, ambientales, de dolor, preguntas 1 y 2 y el resultado final de la calidad de vida, especialmente el dominio físico ( $p=0.009$ ). Conclusión: Después del tratamiento de la YNSA fue posible observar una reducción significativa del dolor. Debido a la mejora del dolor, también fue posible observar y demostrar la mejora de los dominios que componen la calidad de vida.

DESCRIPTORES: Docentes de Enfermería, Educación en Enfermería, Graduación en Auxiliar de Enfermería, Enfermería, Docentes.

RECEBIDO EM: 10/06/2020 APROVADO EM: 11/06/2020

\section{Luana Borges Dutra}

Enfermeira. Mestre em Enfermagem pela Universidade Federal do Estado do Rio de Janeiro (UNIRIO); Pós Graduada em Acupuntura pela UNIBAHIA.

ORCID: 0000-0002-6132-3255

\section{Raphael Neves Barreiros}

Enfermeiro. Mestre em Enfermagem pela Universidade Federal do Estado do Rio de Janeiro (UNIRIO); Pós Graduado em Acupuntura pela UNIBAHIA.

ORCID: 


\section{Caroline de Araújo Mendes}

Enfermeira. Mestre em Enfermagem pela Universidade Federal do Rio de Janeiro (UNIRIO). ORCID:

\section{Joice Alves Cabral}

Enfermeira. Mestre em Enfermagem pela Universidade Federal do Rio de Janeiro (UNIRIO). ORCID:

\section{Carlos Roberto Lyra da Silva}

Enfermeiro, Pós-Doutor pelo Programa Associado de Pós-Graduação em Enfermagem da UPE/UEPB. Doutor em Enfermagem pela Universidade Federal do Rio de Janeiro (UFRJ). Professor Associado do Departamento de Enfermagem Fundamental da Escola de Enfermagem Alfredo Pinto - DEF/EEAP.

ORCID:

\section{Thiago Quinellato Louro}

Enfermeiro, Pós Doutor em Enfermagem, Doutor em Ciências - PPGENFBIO/UNIRIO. Professor Adjunto II do Departamento de Enfermagem so Instituto de Humanidades em Saúde da Universidade Federal Fluminense (UFF). Coordenador do Grupo de Pesquisa: Laboratório de Pesquisa e Experimentação para o Ensino e Cuidado em Enfermagem - L@PETECEnf / UFF. ORCID:

\section{INTRODUÇÃO}

A qualidade de vida $(\mathrm{QV})$ é um tema que atualmente é discutido e avaliado em diversos grupos populacionais, porém ainda há fatores que necessitam ser estudados, para então, pensar em novas estratégias em saúde, intervenções direcionadas, mudanças no estilo de vida e, consequentemente, sua melhoria $^{(1)}$. Entende-se por qualidade de vida a percepção do indivíduo, de sua posição na vida, no contexto da cultura e sistema de valores nos quais ele vive e em relação aos seus objetivos, expectativas, padrões e preocupações ${ }^{(2)}$. Ela abrange diversos fatores, como os citados acima, além da percepção da situação de saúde.

Contudo, sabe-se que um terço do dia é destinado ao trabalho, o ambiente e as atividades desenvolvidas tornam-se fatores de risco ou de proteção para o desenvolvimento de agravos em saúde. $\mathrm{O}$ trabalho pode ser percebido como fonte de felicidade, reconhecimento, realização e prazer, evidenciado pelo progresso pessoal e econômico e também como causador de desgastes físicos e ou psíquicos ${ }^{(3)}$.

As doenças crônicas não transmissíveis são o principal problema de saúde no Brasil, que afeta principalmente a população pobre e os grupos mais vulneráveis. As doenças crônicas possuem diversas causas, que variam com o tempo, hereditariedade, estilo de vida, exposição a fatores ambientais e fisiológicos, iniciam-se e evoluem lentamente. Têm período de duração superior a três meses e, em alguns casos, tendem a se apresentar de forma definitiva e permanente ${ }^{(1)}$.

$\mathrm{O}$ profissional afetado por qualquer tipo de dor pode ter dificuldade em realizar seu papel de forma eficiente e eficaz, com o consequente reflexo na sua vida pessoal no qual reduz significativamente a qualidade de vida. Nesse sentido, é essencial coordenar ações para promover a saúde dos trabalhadores como um mecanismo para melhorar os cuidados de saúde em geral ${ }^{(3)}$.

Estudos afirmam que quando ocorre déficit físico, a incapacidade funcional pode prejudicar a independência e autonomia dessas pessoas, condições que comprometem além da saúde, a $Q V^{(4)}$.

Há vários fatores que interferem na qualidade de vida dos trabalhadores, mais especificamente nos profissionais de saúde, dentre eles: a carga horária semanal de trabalho, a idade, sexo, o regime de dedicação (tempo integral, dedicação parcial e horista), titulação, qualidade dos serviços oferecidos, satisfação com a equipe, relacionamento no serviço, condições de trabalho, turno de trabalho, dentre outros, os quais afetam os domínios psicológicos, relações sociais, aspectos espirituais/religiosidade/ crenças pessoais, meio ambiente e físico ${ }^{(4,5)}$.
Visto isso, acredita-se que o desenvolvimento de estratégias que propiciem o bem-estar dos profissionais de saúde é importante para que possam oferecer excelência no atendimento ao paciente. Devido a essa problemática, busca-se por intervenções dirigidas aos profissionais, mais especificamente à equipe de enfermagem, para reduzir o risco de problemas devido à saúde ocupacional ${ }^{(6)}$.

Torna-se oportuno, após a discussão anterior, delimitar como objetivo: descrever as repercussóes da Nova Craniopuntura de Yamamoto na dor e qualidade de vida de profissionais de saúde.

\section{METODOLOGIA}

Trata-se de um estudo do tipo antes e depois, que implica na realização de uma intervenção, entretanto, diferentemente dos estudos experimentais, não exigindo a randomização dos sujeitos da pesquisa, não havendo grupo controle ${ }^{(7)}$.

Os sujeitos foram 07 profissionais que trabalhavam em uma Estratégia de Saúde da Família (ESF), de um município situado na Região da Baixada Litorânea, do Estado do Rio de Janeiro, de um universo de 17 que trabalhavam na referida unidade, entre diferentes categorias profissionais (agentes de saúde, enfermeiro, técnicas de enfermagem, médico, secretária, serviços gerais, es- 
tagiário - arquivo), sendo os que se enquadraram nos critérios de inclusão e aceitaram participar do estudo, a partir da assinatura do Termo de Consentimento Livre e Esclarecido (TCLE). Tal fato corrobora com os preceitos da Resolução n. ${ }^{\circ}$ 466/2012 de Diretrizes e Normas Regulamentadoras de Pesquisa Envolvendo Seres Humanos do Conselho Nacional de Saúde - CNS ${ }^{(8)}$.

Vale destacar que esta pesquisa foi cadastrada na Plataforma Brasil, onde consta estar devidamente apreciada e aprovada no dia 21 de junho de 2016 pelo Comitê de Ética em Pesquisa da Universidade Federal Fluminense através do CAAE ${ }^{\circ}$ : 56278016.0.0000.5243. A intervenção iniciou no dia 22 de junho de 2016, logo após aprovação do Comitê de Ética e Pesquisa, e seu término se deu no dia 22 de julho de 2016.

Foram estabelecidos como critérios de inclusão: ser profissional de uma ESF e ter alguma queixa de dor.

Para melhor avaliação da Qualidade de Vida e aplicabilidade do instrumento WHOQOL-BREF, o processo de coleta dos dados ocorreu antes de iniciar e após

\section{Quadro 1. Teste de hipótese de Wilcoxon. Rio de Janeiro, RJ, Brasil, 2016}

\begin{tabular}{|l|l|}
\hline WILCOXON & P \\
\hline Dor antes & $0.009^{*}$ \\
\hline Dor depois & $0.009^{*}$ \\
\hline QV antes & $0.02^{*}$ \\
\hline QV depois & $0.009^{*}$ \\
\hline Pergunta 1 antes & $0.009^{*}$ \\
\hline Pergunta 1 depois & $0.009^{*}$ \\
\hline Pergunta 2 antes & \\
\hline Pergunta 2 depois & 0,17 \\
\hline Físico antes & \\
\hline Físico depois & $0.02^{*}$ \\
\hline Psicológico antes & \\
\hline Psicológico depois & \\
\hline Social antes & \\
\hline Social depois & \\
\hline Ambiente antes & \\
\hline Ambiente depois & \\
\hline Nota: $*$ valordep, para ser considerável deve ser $\leq 0,05$. & \\
\hline
\end{tabular}

o término de um tratamento de Nova Craniopuntura de Yamamoto (YNSA) realizado em um período de 4 semanas sendo aplicada 2 sessóes de craniopuntura por semana, estas em dias distintos, totalizando 8 sessões. Esta estratégia vai ao encontro àquela demonstrada em um estudo intitulado "Avaliação do controle da dor miofascial crônica em cabeça e pescoço utilizando a técnica Nova Crânio Acupuntura de Yamamoto em acompanhamento durante 8 semanas", onde obteve-se bons resultados ${ }^{(9)}$.

Após a devida obtenção dos dados, os mesmos foram compilados e analisados com auxílio do programa Bioestat 5.3, com licença gratuita disponibilizada on-line. Os resultados foram apresentados descritivamente, através de medidas de tendência central, frequência simples e o teste de hipóteses, onde foi realizado o teste de Wilcoxon (não paramétrico) em decorrência da amostra $(n=7)$.

\section{RESULTADOS}

Dos 17 profissionais alocados no cenário do estudo, 09 não puderam participar 
Quadro 2. Classificação dos domínios do WHOQOL-BREF, antes e após o tratamento. Rio de Janeiro, RJ, Brasil, 2016

\begin{tabular}{|c|c|c|c|c|}
\hline & DOMÍNIOS & MÉDIA & DESVIO PADRÃO & CLASSIFICAÇÃO \\
\hline \multirow{5}{*}{ 苞 } & Físico & 2,8 & 0,7 & Necessita melhorar \\
\hline & Psicológico & 3,4 & 0,4 & Regular \\
\hline & Relações Sociais & 3,1 & 0,7 & Regular \\
\hline & Meio ambiente & 2,5 & 0,5 & Necessita melhorar \\
\hline & Total & 3 & 0,4 & Regular \\
\hline \multirow{5}{*}{$\frac{n}{0}$} & Físico & 4,3 & 0,4 & Boa \\
\hline & Psicológico & 3,9 & 0,5 & Regular \\
\hline & Relações Sociais & 3,3 & 0,5 & Regular \\
\hline & Meio ambiente & 2,8 & 0,3 & Necessita melhorar \\
\hline & & 3,6 & 0,9 & Regular \\
\hline
\end{tabular}

Quadro 3. Análise das Questões 1 e 2 do WHOQOL-BREF, antes e após o tratamento. Rio de Janeiro, RJ, Brasil, 2016

\begin{tabular}{|c|c|c|c|c|c|}
\hline & QUESTÕES & MÉDIA & MODA & DESVIO PADRÃO & CLASSIFICAÇÃO \\
\hline \multirow{2}{*}{ 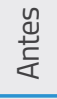 } & Questão 1 & 2,80 & 3,00 & 1 & Necessita melhora \\
\hline & Questão 2 & 2,40 & 2,00 & 0,9 & Necessita melhora \\
\hline \multirow{2}{*}{$\begin{array}{l}\frac{n}{0} \\
\frac{0}{2}\end{array}$} & Questão 1 & 3,8 & 4 & 0,3 & Regular \\
\hline & Questão 2 & 4,5 & 5 & 0,7 & Boa \\
\hline
\end{tabular}

quais sejam: "Como você avaliaria sua qualidade de vida?”, "Quão satisfeito você está com a sua saúde?”. Tal fato se dá pela não contabilização destes dois itens para a média final e a consequente estratificação da classificação da $\mathrm{QV}^{(2)}$.

Avaliando os dados expostos, pode-se afirmar que houve aumento significativo nas médias de ambas as questões, principalmente na questão 2 , onde a média praticamente dobrou (de 2,40 foi para 4,5). Outro dado possível de observar na tabela é a moda de cada questão, na última questão houve aumento surpreendente, de 2 que significa insatisfeito, passou para 5 , escore máximo do WHOQOL- BREF, que significa muito satisfeito.

Como já visto anteriormente, não foi possível observar melhora significativa na classificação geral dos domínios, discutidos no Quadro 3, porém observou-se um aumento nas médias de todos os domínios.

\section{DISCUSSÕES}

O percentual significativo de profissionais que sentem dor, vai de encontro com outras pesquisas, por afirmar que o trabalho implica à exposição de fatores que podem levar a doenças ou ao sofrimento, fato decorrente da própria natureza do ofício e de sua organização. A QV dos trabalhadores está diretamente ligada a fatores, como: horas semanais de trabalho, regime de dedicação, qualidade dos serviços oferecidos, satisfação com a equipe e condições de trabalho ${ }^{(4,5)}$.

Quando se foca no profissional que exerce atividades no âmbito da saúde, alguns fatores são adicionados, pelo próprio objeto de trabalho, como exemplo a dor, sofrimento e a maneira como é organizado seu exercício profissional podem ser prejudiciais ao trabalhador. Por isso a importância de elaborar medidas corretivas e preventivas para melhoria da capacidade laboral, com repercussões na qualidade de vida ${ }^{(10,11)}$.

Os dados relacionados ao gênero são semelhantes a outros estudos, onde o gênero feminino foi o mais prevalente nas instituições de saúde. Vale ressaltar que a maior parte das mulheres também exerce trabalhos domésticos, denotando assim uma possível dupla jornada, que resulta uma sobrecarga de trabalho, evidenciada pelo desgaste físico e mental(10,12-14).

Quando voltamos a discussão para as dores referidas pelos profissionais, é possível afirmar que outros estudos demonstram resultados similares. A prevalência de sintomas osteomusculares evidenciada, pelo seu estudo em trabalhadores de enfermagem, foi de $91,81 \%$, tais sintomas osteomusculares são responsáveis por afastamentos laborais ${ }^{(15)}$. Dentre os que mais acometem a equipe de enfermagem estão as regiôes corporais: pescoço, ombro, lombar, dorsal, tornozelos/ pés, quadris/ coxas. A dor de cabeça também é expressa em outro estudo, onde é possível observar a relação do estresse, a diminuição da qualidade de vida e a redução da capacidade laboral, devido a cefaleia ${ }^{(6)}$.

Por conseguinte, é possível dizer que após o tratamento da craniopuntura, a diminuição da dor foi consideravelmente positiva, o que certamente influenciou na melhora das variáveis citadas acima, tendo em vista que diversos estudos correlacionam a dor dos profissionais de saúde com a diminuição da qualidade de vida ${ }^{(11-16)}$.

Assim sendo, podemos dizer que com a diminuição da dor é possível melhorar diretamente o domínio físico, ao mesmo tempo, os resultados apresentados também nos sugerem que a redução da dor favorece uma melhoria na percepção da qualidade de vida nos domínios psicológico e ambiental.

Outra pesquisa relevante observou que quanto maior o número de condições crônicas, menores são os valores dos domínios da escala de qualidade de vida, onde os domínios mais afetados foram os aspectos físicos e emocionais ${ }^{(1)}$. Com relação aos aspectos emocionais e mentais, um estudo nos trouxe considerações distintas, se comparadas aos autores discutidos até o momento e aos dados encontrados em nossa pesquisa, pois afirma que os escores dos domínios referentes à saúde mental e aspectos emocionais encontrados em seu estudo, 
demonstram que os profissionais de saúde possuíam indicadores de QV suficientes para atuar na rotina assistencial ${ }^{(14)}$.

Logo, há aspectos negativos quanto ao estresse no trabalho e ao suporte social, verificou-se que $85 \%$ dos profissionais considera o apoio social recebido muito insatisfatório, com dificuldades nas relações interpessoais ${ }^{(17)}$. Dados estes que são semelhantes aos encontrados na ESF.

\section{CONCLUSÃO}

Enfim, pode-se dizer que após o trata- mento de craniopuntura, considerada uma técnica de baixo custo, foi possível observar diminuição significativa da dor, comprovada estatisticamente. Em virtude da melhora da dor também foi possível observar e comprovar a melhora de diversas facetas que compõem a qualidade de vida.

Pode-se dizer que algumas dificuldades/ limitações foram encontradas, como por exemplo o número reduzido de profissionais participantes da pesquisa, a dificuldade de ter um ambiente reservado para aplicar os questionários e realizar o tratamento, além de não ter estudos sobre a avaliação da qualidade em profissionais de Estratégia Saúde da Família.

Após a realização deste estudo fica evidente a necessidade de intervenções voltadas para os profissionais de saúde da atenção primária. Intervenções estas que visem conhecer, prevenir e intervir nos domínios mais afetados, bem como diminuam as queixas de dor, sejam estas causadas ou não pela atividade laboral. Com o propósito de melhorar a QV do profissional dentro e fora do ambiente de trabalho, aumentando, consequentemente, a satisfação dos usuários pelos serviços prestados.

\section{REFERÊNCIAS}

1. Saraiva LEF, Medeiros LP, Melo MDM, Tiburcio MP, Costa IKF, Torres GV. Condições crônicas de saúde relacionadas à qualidade de vida de servidores federais. Rev Gaúcha Enferm. [Internet]. 2015 June [citado 2019 Set 19];36(2):35-41. http://dx.doi.org/10.1590/19831447.2015.02.46666.

2. World Health Organization (CH). The development of the World Health Organization quality of life assessment instrument (WHOQOL). In: Orley J, Kuyken W, editors. Quality of life assessment: international perspectives. Berlin: Springer Verlag. 1994. p. 41-60.

3. Santos Carneiro TM, Araújo Cordeiro, TM de. Capacidade para o trabalho entre trabalhadores de enfermagem da atenção básica à saúde. Bahia, Brasil. Rev. salud pública. 2018 Ago ;20(4):422-429. http://dx.doi.org/10.15446/rsap.v20n4.53568.

4. Marcacine, PR et al. Qualidade de vida, fatores sociodemográficos e ocupacionais de mulheres trabalhadoras. Ciência \& Saúde Coletiva. 2019, v.24,n.3,pp.749-760. doi: https://doi.org/10.1590/141381232018243.31972016.

5. Souza SBC, Tavares JP, Macedo ABT, Moreira WP, Lautert L. Influência do turno de trabalho e cronotipo na qualidade de vida dos trabalhadores de enfermagem. Rev Gaúcha Enferm. 2012; 33(4):7985. http://dx.doi.org/10.1590/S1983-14472012000400010.

6. Happell B, Dwyer T, Reid-Searl K, Burke KJ, Caperchione CM, Gaskin CJ. Nurses and stress: recognizing causes and seeking solutions. J Nurs Manag. 2013 May;21(4):638-47. doi: https://doi. org/10.1111/jonm.12037.

7. Polit DF, Beck CT. Fundamentos da pesquisa em enfermagem. 7. ed. Porto Alegre: Artmed; 2011.

8. Ministério da Saúde, Conselho Nacional de Saúde (BR). Resolução n. ${ }^{\circ}$ 466, de 12 de dezembro de 2012 . Diretrizes e normas regulamentadoras de pesquisas envolvendo seres humanos. Diário Oficial da União da República Federativa do Brasil. 2013 jun 13;150(112 Seção 1):59-62.

9. Correia LM, Alberti D, Lopes SS. Avaliação do controle da dor miofascial crônica em cabeça e pescoço utilizando a técnica Nova Crânio Acupuntura de Yamamoto em acompanhamento durante 8 sema- nas. Rev Dor. São Paulo, 2015 abr-jun; 16(2):81-5. doi: http://dx.doi. org/10.5935/1806-0013.20150016.

10. Souza et al. Capacidade para o trabalho e sintomas osteomusculares em trabalhadores de um hospital público. Fisioter Pesq. 2015;22(2):182-190. doi: http://dx.doi.org/10.590/18092950/14123722022015.

11. Vidor C da R, Mahmud MAl, Farias LF, Silva CA, Ferrari JN, Comel JC, et al. Prevalência de dor osteomuscular em profissionais de enfermagem de equipes de cirurgia em um hospital universitário. Revista Acta Fisiátrica. 2014;21(1):6-10. doi: https://doi. org/10.5935/0104-7795.20140002.

12. Horta PM, Cardoso AH, Lopes ACS, Santos LC. Qualidade de vida entre mulheres com excesso de peso e doenças crônicas não transmissíveis. Rev. Gaúcha Enferm. 2013 Dec; 34(4):121-129. doi: http://dx.doi.org/10.1590/S1983-14472013000400016.

13. Stefane T, Santos AM, Marinovic A, Hortense P. Dor lombar crônica: intensidade de dor, incapacidade e qualidade de vida. 2013; 26(1):14-20. doi: http://dx.doi.org/10.1590/S010321002013000100004.

14. Oliveira RC, Santos JN, Rabelo ATV, MAGALHÃES MC. Ruído e trabalhadores de Unidades de Suporte Móveis. CoDAS 2015;27(3):215-22. 2015; 27(3):215-222. doi: http://dx.doi. org/10.1590/2317-1782/20152014136.

15. Moreira MM, Marcondes C, Geremia DS. Padrões de sono entre profissionais da enfermagem. Revista de Atenção à Saúde, v. 13, no 44, ar./jun. 2015, p.11-16. doi: https://doi.org/10.13037/ras. vol13n44.2493.

16. Brettschneider C, Leicht H, Bickel H, Dahlhaus A, Fuchs A, Gensichen J. et al. Relative impact of multimorbid chronic conditions on health-related quality of life: results from the MultiCare Cohort Study. PLoS One. 2013 ;8(6):e66742. doi: https://doi.org/10.1371/ journal.pone.0066742.

17. Silva AM, Guimarães LA. Occupational Stress and Quality of Life in Nursing. Paidéia jan-apr. 2016, Vol.26, No.63,63-70. doi: http:// dx.doi.org/10.1590/1982-43272663201608 